Página inicial: 61 - Página final: 74

TIPO DE ARTÍCULO: de Investigación

\title{
EL TRASFONDO ECONÓMICO DE UN CRIMEN DE LESA HUMANIDAD
}

THE ECONOMIC BACKGROUND OF A CRIME AGAINST HUMANITY

Recibido: Abril 2013 Revisado: Octubre 2013 Aceptado: Diciembre 10 de 2013

Por: Renán Vega Cantor ${ }^{1}$.

\section{RESUMEN.}

El presente artículo resultado de investigación, pretende aportar elementos clave, que den cuenta de los nexos entre libre comercio y la violación permanente de los derechos humanos e incluso con delitos de lesa humanidad; situaciones que no se constituyen en hechos aislados, circunstanciales y producto de la simultaneidad aparente entre delincuencia en abstracto, desarrollada en zonas donde se proyectan y desarrollan proyectos estratégicos para la economía global, sino en un asunto relacionado, provocado, sostenido por los grupos económicos, políticos tanto nacionales como internacionales que ganan con estos mega-proyectos.

\section{PALABRAS CLAVE.}

imperialismo, desarrollo, neoliberalismo, américa latina, bio-geopolítica.

\begin{abstract}
.
This article, as a result of research, aims to provide key elements, which take into account the links between the free trade and the permanent violation of the human rights and even with crimes against humanity; situations that do not constitute themselves in isolated, circumstantial facts, which are the product of the apparent simultaneousness between crime in abstract, developed in areas where strategic projects for the global economy are projected and developed, but in a matter related, caused, sustained by both national and international economic, political groups that make a profit with these mega-projects.
\end{abstract}

\section{KEY WORDS.}

Imperialism; Development; Neoliberalism; Latin America; and Bio-geo-politics.

\footnotetext{
${ }^{1}$ Historiador. Profesor titular de la Universidad Pedagógica Nacional, de Bogotá, Colombia. Autor y compilador de los libros Marx y el siglo XXI (2 volúmenes), Editorial Pensamiento Crítico, Bogotá, 1998-1999; Gente muy Rebelde, (4 volúmenes), Editorial Pensamiento Crítico, Bogotá, 2002; Neoliberalismo: mito y realidad; El Caos Planetario, Ediciones Herramienta, 1999; entre otros. Premio Libertador, Venezuela, 2008. Su último libro publicado es Capitalismo y Despojo, Editor Revista CEPA. Colombia_carajo@hotmail.com, renan.vega@padagogica.edu.co
} 


\section{Introducción.}

EL discurso neoliberal y la propaganda que difunde desde hace décadas afirman que el libre comercio es sinónimo de prosperidad y bienestar para los países que lo adopten. En el discurso convencional y dominante, que incluso han asumido sectores de la izquierda llamada "light" a nivel mundial, se asegura que la apertura comercial de un país le traerá desarrollo y bienestar, "ríos de leche y de miel". Para completar el cuadro, al evocar los "milagros del libre comercio" se afirma que son un resultado de las fuerzas irreversibles del mercado que se imponen por su superioridad intrínseca sobre sus "enemigos naturales", entre los que se encuentran el Estado, los sindicatos, las organizaciones sociales, fuerza campesina, indigenas, organizaciones populares, etc.

Estas afirmaciones que sustentan el discurso neoliberal justifican la imposición de Tratados de Libre Comercio después de 1994, por parte de la mayoría de países de América Latina, con los que entrariamos en el Primer Mundo, porque se superarian siglos de aislamiento secular, que nos habian mantenido al margen de la modernización y el progreso. Con una lógica bastante primaria, propia de los economistas neoliberales, a rajatabla se impusieron y siguen imponiendo hoy los TLC.

Aunque las evidencias empíricas -como en el caso de México- indiquen un impacto negativo sobre la vida de la población, los librecambistas repiten el estribillo que el libre comercio representa la redención para quienes "valientemente" lo adopten en forma consciente porque, se agrega, que no existe alternativa: se trata de "exportar o morir".

Quienes esto sostienen no están muy equivocados, salvo que haya que darle la vuelta al dilema, porque en realidad esa consigna devino en exportar y morir o, más precisamente, morir para exportar, exportar aunque muchos mueran, exportar gracias a la muerte de miles de personas, o exportar sobre los huesos de los muertos.

Esto es indispensable recordarlo, porque el libre comercio debe entenderse como otra forma de guerra, como lo decía Bertolt Brecht: "He oído a mucha gente decir que el comercio y la economía son humanos, y que sólo la guerra es inhumana. Pero resulta que, en primer lugar, ni el comercio ni la economía son humanos, y en segundo lugar, nos conducen a la guerra [...] La barbarie procede de la barbarie, puesto que la guerra procede de la economía" (Brecht, 1994)

Estas macabras características del libre comercio han sido estudiadas en forma magistral en La Doctrina del Shock, la obra de Naomi Klein, en el cual se demuestra con numerosos ejemplos que la libertad de mercado no es un resultado de la mano invisible del mercado, sino del brazo bien armado de diversas fracciones del capital que propician la guerra, la tortura y la muerte de millones de seres humanos. El libre mercado se impone sobre una interminable pila de cadáveres de gente pobre y humilde se levantan las exitosas economías exportadoras, siendo Chile el ejemplo más alabado.

El descubrimiento analítico de Naomi Klein puede sintetizarse con pocas palabras: para que el libre comercio opere, sin incómodos obstáculos sociales, los capitalistas de cada país generan un estado previo de pánico y terror, que inmoviliza a la población, posibilitando la apertura comercial y la aplicación de medidas neoliberales. En otros términos, existe una estrecha relación entre libre mercado y tortura, porque esta última es uno de los instrumentos favoritos para aterrorizar a 
la población y consolidar las economias de exportación, que luego son presentadas como modelos exitosos y ejemplos que deben ser imitados.

Sobre los trabajadores, campesinos, indigenas, afrodescendientes, mujeres humildes y, en general, habitantes pobres del campo y la ciudad, se erigen los monumentos del mercado libre.

"Si recordamos que algunas de las violaciones de derechos humanos más despreciables de este siglo, que hasta ahora se consideraban como actos de sadismo fruto de regimenes antidemocráticos, fueron de hecho un intento deliberado de aterrorizar al pueblo, y se articularon activamente para preparar el terreno e introducir las 'reformas' radicales que habrian de traer ese ansiado libre mercado" (Klein, 2007).

Si se trata de buscar una ilustración práctica de los nexos entre libre comercio, tortura y muerte, Colombia es el modelo más brutal, como lo ejemplifica lo que sucede por estos días, y desde hace varios años, en el Puerto de Buenaventura, en el Océano Pacífico. Sin embargo, no debe suponerse que este es un hecho aislado, circunstancial y producto de la delincuencia o de los "violentos" en abstracto, como suele presentarlo los medios masivos de comunicacón. De ninguna manera, el Puerto de Buenaventura es sólo una muestra a pequeña escala del terror que acompaña la imposición de casi dos decenas de Tratados de Libre Comercio entre Colombia y el resto del mundo.

Por otra parte, como se concluye de la contundente realidad, que se reflejan en muchos estudios sobre víctimas y paramilitarismo en Colombia, el símbolo criminal que identifica más claramente la apertura comercial de Colombia es la motosierra, que de ser un medio de trabajo utilizada para cortar árboles y madera, en manos de los paramilitares -ligados en forma directa al Estado colombiano y financiados y armados por las clases dominantes, políticos nacionales, regionales, ganaderos, industriales colombianos y empresas transnacionales- se transformó en el más horroroso y cruel instrumento de tortura y de muerte, por medio del cual se procede a desmembrar vivas a las personas, se les desmembra (pica) y luego se les bota como residuos a un caño, a una ciénaga, al mar o se les entierra en fosas comunes (Balbin \& Insuasty Rodriguez, 2009).

En todos los lugares del territorio colombiano que hoy son presentados como modelos de éxito exportador, como paso previo se destruyeron las sociedades y economías locales, al tiempo que se arrasaban las bases sociales de los movimientos reivindicativos y de la insurgencia, mediante el terror planificado.

\section{Metodologia.}

Para el desarrollo del presente texto, se consideran cuatro cuestiones:

- En primer lugar, se indica cuáles son las características de la guerra mundial por los recursos traducido en proyectos estratégicos de desarrollo.

- En segundo lugar, se subrayan los aspectos medulares de la estrategia de control territorial como proyectos a mediano plazo, que dan tramite a la implementación de grandes proyectos para el sector productivo internacional; 
- En tercer lugar, el impacto de dichas estrategia de control territorial especificando casos específicos enfatizando en concreto, el caso del puerto de Buenaventura - Colombia.

Para ello se acuden a fuente oficiales, centros de documentación e investigación, contrastación, análisis de fuentes.

\section{Resultado - discusiones.}

El método más frecuente, el que puede denominarse como la contribución auténticamente colombiana a la historia universal de la infamia, ha sido el de la motosierra para destrozar a seres humanos, como se ilustra en la película, Perro come perro. No por azar, la BACRIM (Banda Criminal) más sanguinaria, la de los Urabeños y asociada al Expresidente Alvaro Uribe Vélez, acuñó como lema de guerra (electoral y militar) el de Mano firme, Motosierra grande. (El Tiempo, 2014)

En Medellín, la "tasita de plata" de los traquetos paisas y de las clases dominantes de Colombia paramilitares la emplearon y sobre miles de muertos de esta ciudad y de las zonas circundantes se levanta el proyecto de una ciudad competitiva, turística y abierta a las inversiones extranjeras. (Franco Restrepo, 2011)

En la costa atlántica, durante años se efectuó una ronda de muerte a punta de machete y motosierra, en muchas ocasiones acompasada por el ritmo de gaitas y vallenatos y en esos mismos territorios se erigen megaproyectos, represas, grandes haciendas y centros comerciales, que se enaltecen como los milagros de la "paz paramilitar".

Para no ir muy lejos, a la ciudad de Monteria, capital de Córdoba, una de las cunas del paramilitarismo, y sitio de donde es oriundo Salvatore Mancuso (Revista Semana, 2011) -un paramilitar aristocrático y bestial- se le compara con Miami y, lo que parece un chiste cruel, fue distinguida como una de las cincos ciudades más sostenibles del planeta en el año 2014, por parte del Fondo Mundial para la Naturaleza (E1 Espectador, 2014).

Los Llanos Orientales han sido la cuna de ejércitos paramilitares, escenario a vasta escala del exterminio de la Unión Patriótica y donde se efectuaron masacres, como la de Mapiripan en 1997, con participación de los Estados Unidos y las Fuerzas Armadas de Colombia. Es a ese mismo territorio de los Llanos al que los capitalistas locales y foráneos quieren convertir en un emporio agrícola de vocación exportadora que produzca caña, palma, caucho y materias primas para generar agrocombustibles, y en el que se extraen miles de barriles de petróleo por la Pacific Rubiales y otras compañias que, como se observa en estos momentos, destruyen las reservas hídricas de algunos sectores del Departamento del Casanare.

Se podrian seguir enumerando en forma rutinaria las regiones de Colombia que aparecen como emblemas del libre comercio, todas las cuales tienen un pasado y un presente pleno de crímenes y torturas, que se constituye en el telón de fondo en el que, en medio del terror de la motosierra, se despejaron territorios, se asesinaron a sus lideres sociales, se masacraron habitantes lugareños y otros fueron obligados a huir... Y luego vinieron los "prósperos" y "honorables" hombres de empresa a fortalecer las empresas exportadoras. Esto lo manifestaron sin pestañear, como si fueran laureados economistas de encopetadas universidades, los jefes paramilitares. Por ejemplo, Vicente Castaño afirmó en una ocasión: 
"Queremos que nos dejen hacer nuevos modelos de empresas que ya hemos venido desarrollando a nivel nacional. [...] En Urabá tenemos cultivos de palma. Yo mismo conseguí los empresarios para invertir en esos proyectos que son duraderos y productivos. La idea es llevar a los ricos a invertir en ese tipo de proyectos en diferentes zonas del país. Al llevar a los ricos a esas zonas llegan las instituciones del Estado. Desafortunadamente las instituciones del Estado sólo le caminan a esas cosas cuando están los ricos. Hay que llevar ricos a todas las regiones del país y esa es una de las misiones que tienen todos los comandantes" (Revista Semana, 2005).

En estas afirmaciones no puede dejarse pasar un detalle fundamental:

En Urabá, la región a la que se refiere Vicente Castaño, se presentó un proyecto de limpieza social por los paramilitares, los militares y el Estado, que recurrió al terror y a las masacres. No por casualidad de ese proyecto regional salió un político local directamente a la presidencia de la República. Y los productos estrellas de ese proyecto de muerte, el banano y la palma aceitera (o palma africana, por su origen geográfico), se consolidaron como renglones exportadores del país gracias a la motosierra. Luego de que miles de trabajadores fueran asesinados, se destruyeran sus sindicatos clasistas, se exterminara a la Unión Patriota, la Chiquita Brands financiara a las bandas de paramilitares, se "pacificó" la región y ésta se convirtió en una zona competitiva en la producción y exportación del banano, por supuesto un banano sangriento. En cuanto a la palma, comunidades afrodescendientes fueron masacradas para que en sus tierras se iniciara el proyecto terrateniente de trasformar el país en la Malasia de Sudamérica, sembrando la región con la "palma de la muerte" (O Loingsigh, 2013).

El paramilitar conocido como El Alemán pontificaba sobre las bondades del librecomercio, como cualquier Ministro de Hacienda o Agricultura formado en las "mejores escuelas de negocios" del país o del mundo, cuando sostenía:

"Queremos [...] un desarrollo regional que desde grandes proyectos de infraestructura posibilite el establecimiento de cadenas productivas y de comercialización que aprovechen las ventajas geoestratégicas de nuestro país, generando una verdadera revolución constructiva y democratizadora del agro que integre a la industria y al gran capital con el trabajo asociado y con el pequeño propietario rural" (Contreras, 1999).

Quien no tenga ni idea de quien procede esta afirmación puede pensar que nos encontramos ante un consultor internacional del Banco Mundial o ante un ilustre economista de Chicago, pero lo llamativo es que quien así hablaba era el cabecilla del bloque paramilitar Elmer Cárdenas, responsable de la muerte de miles de campesinos en Urabá. Este mismo individuo, para más señas, controlaba el negocio de la madera en el Atrato, e impulsó el Plan Motosierra que se sustentaba en poseer 200 motosierras y 500 mulas, que se usaban para presionar a los campesinos y obligarlos a producir grandes cantidades de madera, mediante la aparcería y el endeude forzado: "Le vendiamos al que nos trajera la plata en efectivo, no fiábamos. Nos llegaba la plata en costal. Había meses de 200 o 100 millones (de pesos). Era la participación que teníamos. Y el campesino tenía la posibilidad de volver al monte e ir pagando la herramienta que no tenia". Como en cualquier enclave, "a cambio de las herramientas, los campesinos empeñaban la madera cortada para pagar la deuda y el resto [...] se les pagaba con vales que sólo podian redimir en negocios controlados por los "paras"' (Verdad Abierta, 2014). 
Tan jugoso negocio no podía prosperar sin acudir a la fuerza bruta, porque al bloque Elmer Cárdenas fue responsable del "episodio de un grupo de paras que luego de cortar la cabeza de una de sus víctimas, jugó fútbol con ella" y del "asesinato con sevicia de mujeres embarazadas y la quema de caserios" (Revista Semana, 2006).

Como veremos enseguida, lo que acontece en Buenaventura tiene notables antecedentes y similitudes con lo sucedido en otras regiones de Colombia, en donde se rubricó un nexo orgánico entre el libre comercio y la motosierra. Esto es necesario plantearlo para eludir las explicaciones convencionales de los medios de desinformación, quienes se rasgan las vestiduras y pretenden que los hechos de Buenaventura son inéditos y un resultado exclusivo de la delincuencia y el narcotráfico, sin ninguna relación con los magaproyectos de modernización del puerto. Como si, además, el narcotráfico y la violencia asociada no fueran también expresiones del libre comercio, impulsadas por los Estados Unidos.

En Buenaventura, ubicada a 115 kilómetros de Cali, se encuentra el primer puerto de Colombia, tanto por el volumen de carga que llega del mundo como el que se envía desde nuestro país hacia el exterior. El puerto está enclavado en la región del Choco biogeográfico, un auténtico tesoro de la naturaleza que se extiende desde Panamá hasta el Ecuador en un área de 187 kilómetros cuadrados, siendo la zona más lluviosa del mundo. Su elevada pluviosidad, su ubicación tropical y su aislamiento la convierten en una de las regiones más biodiversas del planeta en plantas, mamíferos, aves, reptiles y anfibios, además de que posee maderas, agua y minerales. Esta riqueza es apetecida por los países imperialistas y sus empresas transnacionales, cuyo interés aumenta por la geoestratégica ubicación, comercial y militar, de Buenaventura.

Junto a esa riqueza de la naturaleza, coexiste una terrible desigualdad social, que exacerba aún más el libre comercio -entre unos pocos ganadores y muchos perdedores-, lo cual ha hecho emerger dos realidades opuestas, dos Buenaventuras que están claramente separadas, como es típico en los enclaves económicos, cuya característica distintiva es la de servir de punto de tránsito de mercancías hacia el mercado mundial. Esas dos Buenaventuras están formadas por el puerto y la ciudad. El puerto se localiza en una envidiable posición geográfica, en el corazón de la cuenca del Pacífico, hoy por hoy el centro del comercio mundial, y próximo a las principales rutas maritimas del planeta, entre ellas el Canal de Panamá. Por ese puerto circula el $60 \%$ del comercio exterior de Colombia, incluyendo el $80 \%$ del café, y está equipado con lo último en tecnología, es limpio y automatizado. Funciona sin parar las 24 horas del dia y un muro electrificado lo separa del resto de la ciudad. Ese puerto es apetecido por inversionistas y transnacionales, y como parte de esa avanzada se construyó el Terminal de Contenedores. Está protegido metro a metro por centenares de miembros de las fuerzas militares del Estado y guardias privados. Se le menciona siempre como modelo exitoso de la integración exitosa del país al comercio mundial.

Por el puerto pasan diariamente enormes buques trasatlánticos que descargan y se llevan cada año doce millones de toneladas, en un típico intercambio desigual puesto que "por cada 10 contenedores que llegan llenos de juguetes, ropa, carros y electrodomésticos de Asia, sólo tres regresan cargados con productos de postre: café, plátanos o azúcar de Colombia" (Weis, 2012). 
El Puerto de Buenaventura es una prueba a pequeña escala de los "beneficios" del libre comercio, que tanto exaltan Mario Vargas Llosa y todos los neoliberales de su estilo. Este puerto aparece como el epicentro de megaproyectos de infraestructura y como la capital colombiana de la Alianza del Pacífico en que participan Chile, México, Costa Rica y Colombia. Es un puerto sin gente, con pocos trabajadores, hecho para descargar containers y almacenar mercancias, porque las grandes grúas y enormes recipientes sustituyeron a los estibadores. Mientras que a finales de la década de 1980 trabajaban en el puerto 2500 trabajadores, en la actualidad la Sociedad Portuaria emplea a 181, quienes garantizan la conexión con el mercado mundial (Almario, 2007).

Al lado del iluminado, limpio, trasparente, privatizado y "pacífico" puerto está la ciudad, en donde viven en la pobreza absoluta 400 mil personas, cuyas condiciones de vida alcanzan tal indignidad que bien podría llamarse Malaventura. Cada cuatro de cinco de sus habitantes, en un $88 \%$ afrodescendientes, son pobres absolutos, el desempleo supera el $60 \%$, el $35 \%$ de la población no cuentan con acceso al agua potable, al alcantarillado, a la energía eléctrica y al gas. Si antes había pobreza ahora lo que hay es miseria extrema, como resultado de la privatización de Colpuertos, puesto que los trabajadores que estaban ligados a esta empresa pública eran quienes redistribuian sus ingresos entre la población, y con sus salarios dinamizaban la economía local. La eliminación de esa empresa y la formación de La Sociedad Portuaria le ocasionaron un golpe mortal a la economía de la ciudad y, en contra de la cartilla neoliberal del libre comercio, propiciaron que Buenaventura se aislara no sólo del mundo sino del resto de Colombia.

Esto ha sido el resultado de la privatización de la actividad portuaria porque se rompió el vínculo entre el Puerto y la ciudad, como consecuencia de lo cual "Buenaventura, negra, mulata, indigena ha devenido gueto y el gueto va camino de convertirse en una gran prisión en la que las fuerzas armadas del Estado supervisan la matanza que ocurre ante sus ojos" (Salazar, 2007). Malaventura crece y se expande para recibir los miles de desplazados de las zonas circundantes, a quienes se les despoja de sus tierras y bienes comunes, con lo que se ahondan los problemas de miseria y desempleo. Nada la une con el puerto, ni negocios, ni trabajo, ni actividad económica, porque la ciudad no transforma ningún producto que provenga del puerto y ninguna empresa de la ciudad abastece al puerto.

Para sobrevivir, como en cualquier prisión, los bonaerenses deben recurrir a lo que está al alcance de la mano, que en este caso es el microtráfico de estupefacientes, pero a un nivel paupérrimo y miserable, que nada tiene que ver con los grandes negocios y fortunas de los capos de los carteles de otras regiones. Se lucha entre pobres para conseguir unos cuantos centavos, con lo cual se completa el círculo vicioso de delincuencia, descomposición del tejido social y abandono de cualquier lucha colectiva. Sin embargo, en Buenaventura no hay dinero, porque éste se encuentra afuera, en las manos de quienes ordenan los envíos, "los dueños de las caletas, los que hacen los negocios con los DEA en Miami o en Nueva York, los que pagan por matar a los muchachos que han participado en algún envío fallido, los que pagan por ejercer un control precario sobre las zonas claves de la ciudad, los que se apropian de los escasos dineros públicos. Por eso, en las calles con nombres de temas de salsa los muertos mueren sin dinero y todos los dineros viven en la pobreza absoluta, sin agua, sin alcantarillas, sin educación, sin esperanza". La miseria y la muerte están ligadas al comercio mundial, porque "los que pagan por matar y los que disfrutan del dinero por cuya causa tantos mueren 
en Buenaventura no están en la ciudad y no viven en los barrios periféricos en los que rondan la muerte y el terror" (Salazar, 2007). Esto sucede, aunque en la vida cotidiana la población pobre de la ciudad (es decir, casi todos sus habitantes) haya sido encarcelada en un gueto, aislado del resto del territorio colombiano, y que no le interesa ni al Estado ni a las clases dominantes.

Para completar el acoso y el agobio, la Armada ha impuesto una estricta vigilancia costera, so pretexto de evitar la entrada y salida de embarcaciones ilegales, y ha prohibido que los pescadores locales salgan a pescar de noche y les raciona la cantidad de combustible que les venden, como consecuencia de lo cual se dispararon los precios de gasolina en el "mercado negro" y se restringió la pesca, uno de los pocas actividades legales que todavía existian. Esto ha originado la cruel paradoja que en Buenaventura, en cuyo mar se encuentra una gran variedad de pesca, sus habitantes compren pescado importado mientras "los barcos pesqueros de Asia arrasan en las aguas profundas frente a la costa. Las pesqueras colombianas en Buenaventura quebraron y sus fábricas se están convirtiendo en almacenes para carbón y otros recursos que esperan su embarque para Asia” (Weis, 2012).

Como para que no queden dudas de que la realidad supera el realismo mágico de Gabriel García Márquez, ja Malaventura se le denomina oficialmente por el Estado como el Distrito Especial, Industrial, Portuario, Biodiverso y Ecoturístico de Buenaventura! ¡Típico en un país de gramáticos y leguleyos que suponen que con nombres rimbombantes se edulcora la miseria y la infamia!

Que estos dos mundos tan cercanos y separados por una inmensa barrera ya no tienen ninguna relación, lo testifica de manera cínica Domingo Chinea, gerente general de la Sociedad Portuaria Regional de Buenaventura, para quien, mientras los pobres son descuartizados en los barrios de Bajamar, el Puerto "está trabajando perfectamente". En el mismo sentido, el gerente de Hamburg Süd, una empresa naviera alemana -una de las veinte más importantes del mundo- que en el 2013 se instaló en el puerto, señala que "el tema (sic) de Buenaventura no ha impactado el comercio y el movimiento de carga de ninguna manera" (Legiscomex.com, 2014). En otras palabras, como lo dijo el Presidente de la Cámara de Comercio local, "Mientras la maquinita de hacer dinero (el puerto) siga facturando, el Estado no atenderá las problemáticas de Buenaventura". Para los promotores del libre comercio en Buenaventura queda claro que una cosa es la gente y otra la carga, es decir, la ganancia.

La gente de Malaventura ya no interesa al capital ni como fuerza de trabajo, ni como consumidores porque no tienen ingresos de ninguna índole, pero al capital si le apetecen los lugares en donde se hacinan esos pobres, sobre todo los barrios de bajamar, porque allí se tienen proyectadas obras de ampliación del puerto y de "modernización económica". Los habitantes de esos lugares son vistos como incómodos estorbos, que impide el progreso del puerto, y a los que se debe quitar del camino, como sea. Por este evidente interés, se libra una guerra brutal contra la gente más pobre, con el objetivo de expulsarla de sus lugares de habitación. Por supuesto, los asesinos intelectuales e ideólogos del mercado libre no se manchan directamente las manos con sangre, sino que actúan por medio de los grupos paramilitares -con la aquiescencia y participación velada del Estado y sus fuerzas represivas- que cambian de nombre (ahora se llaman los Chocoanos, Los Rastrojos, La empresa...,) pero siempre actúan en representación del gran capital colombiano o extranjero, con la finalidad de conseguir los apetecidos terrenos, que luego de ser 
arrebatados o comprados a bajo precio a sus legítimos propietarios son vendidos a los inversionistas en finca raíz y a los agentes de las empresas portuarias.

Las bandas criminales de paramilitares recurren a todos los medios en su propósito de desalojar los barrios de bajamar, por donde se van a construir los megaproyectos. Entre esos medios se encuentran los "incendios de clase" o la "demolición en caliente", como la denomina el geógrafo Mike Davis, que consisten en quemar las casas de los pobres para obligarlos a salir y luego apropiarse de los terrenos ahora yermos y deshabitados (Davis, 2007).

Eso se ha hecho en varias ocasiones, como recientemente, el primero de abril de 2014, cuando fueron devoradas por las llamas 35 viviendas en el Barrio Santa Fe, quedando 196 familias en la intemperie. La gente afectada señaló que "las bandas las quemaron para que nos vayamos del todo". Estas afirmaciones se relacionan con el hecho que "Santa Fe es uno de los barrios de bajamar que han sido codiciados por la administración distrital para adelantar la ampliación portuaria de la Terminal de Contenedores de Buenaventura (TCBuen), ubicada al noroeste de la ciudad. La terminal, que según sus representantes, factura cerca de U\$122.000 millones al año y ha buscado la expansión en los últimos cinco años" (Valenzuela, 2014).

Como sucede en el resto del país, donde los paramilitares actúan tranquila e impunemente, la región está militarizada, sin necesidad de que se la tomen las fuerzas armadas, como lo anunció triunfalmente Juan Manuel Santos, porque a escasos 9 kilómetros se encuentra Bahía Málaga, en donde funciona la principal Base Militar de la Infantería de Marina, y ha hecho presencia la Marina de los Estados Unidos. Sencillamente, la llegada de más tropa lo que quiere es consolidar el proceso de expulsión de los pobres de los barrios de bajamar.

\section{Conclusiones.}

Con lo dicho anteriormente, se quiere resaltar la razón principal que explica el terror generalizado que se ha impuesto desde hace años en Buenaventura, detrás del cual se encuentran los grandes inversionistas nacionales y extranjeros, aunque ellos nunca aparezcan como directos responsables. Dichos inversionistas aplican a las mil maravillas el manual del capitalismo del shock, que ordena aterrorizar a la población para hacer avanzar los proyectos de "desarrollo" y "modernización" propios del capitalismo neoliberal. Algunos habitantes de la ciudad entienden lo que se mueve detrás de los descuartizamientos, como lo indica una dirigente social que prefiere permanecer anónima: "Lo que está en el fondo de esta violencia no es sólo el narcotráfico, es el control territorial del municipio, es una táctica de terror para que la gente se vaya de la zona insular 'para' y se desplace hacia las zonas rurales, para que los megaproyectos puedan tener rienda suelta. Los mafiosos, aliados con algunos empresarios, quieren sacar a la gente a punta de miedo y comprando barato, para luego hacer buenos negocios" (Molano Jimeno, 2013).

Malaventura, la de los pobres, y Buenaventura, el puerto del capital, no figuran en los mismos planes de la Alianza del Pacífico, el plan de Libre Comercio que ha entrado en marcha para que los cipayos de América Latina (Chile, Colombia, México y Costa Rica) le preparen el terreno a las multinacionales de Estados Unidos, en su proyecto de revivir el ALCA. Para esa Alianza del Pacifico, que pretender extraer 
y llevar rápido aquellos productos primarios (petróleo, carbón, madera, minerales, cocaína...) que necesita el capitalismo mundial, lo prioritario es el puerto, no la gente. Por eso ponderan las obras indispensables para que Colombia sea competitiva en el concierto mundial, tales como la doble calzada Buga-Buenaventura, el aumento del tamaño de los muelles, la ampliación de los depósitos de carga y descarga, la construcción de un malecón... Pura cuestión de negocios y de dinero. A ese puerto es el que se quiere declarar la capital colombiana de la Alianza del Pacífico, donde no haya gente y mucho menos si son pobres y afrodescendientes, porque aparte de la dominación de clase impera el racismo.

Malaventura, donde vive la gente y corre la sangre a chorros, se tortura y se procede a desmembrar a jóvenes y mujeres con motosierra, hachas y machetes, porque son un estorbo para los "empresarios de bien", los partidarios del libre comercio. Aún más, su terrorifica miseria, sus casas derruidas, su suciedad no puede ser ni siquiera vista por los grandes inversionistas del mundo. Por esa razón, allí no se reunieron a comienzos de este año los presidentes de la Alianza del Pacífico, que se sentaron a manteles a mil cien kilómetros de distancia, en Cartagena, que no está sobre el Océano Pacífico, sino al otro lado, en el Mar Caribe. Tan inesperados giros geográficos, en una vuelta de tuerca, llevan a que una cumbre del Pacífico se reúna en el Atlántico, lo cual no importa, porque la sapiencia geográfica no es propia de los tecnócratas del Libre Comercio y porque lo fundamental es la buena imagen que el Estado colombiano como huésped les debe mostrar a sus socios comerciales. Además, en Malaventura por la magnitud de la miseria urbana no es posible realizar lo que se ha hecho recientemente en Medellin durante el Foro Mundial Urbano, cuyos pobres fueron sacados de la ciudad durante los ochos días que duró el encuentro, para que los ilustres visitantes no se alteraran al contemplar a mendigos e indigentes, puesto que eso afea el milagro paisa y altera negativamente el buen clima de los negocios (El Espectador, 2014).

Ni siquiera eso de expulsar a los pobres del centro de la ciudad puede hacerse en Buenaventura, porque tendrian que, literalmente desocuparla, lo que no es fácil, ¿por qué a dónde y cómo van a trasladar a 400 mil pobres? ¿Acaso los van a echar al mar, luego de picarlos a todos, para que los inversionistas y presidentes de la Alianza del Pacífico puedan almorzar con tranquilidad algún día en un lujoso hotel de la empobrecida ciudad, sin ver a un pobre ni a un afro en su camino y sin contemplar ni un tugurio? Aunque eso no lo puedan hacer plenamente, por ahora se fortalece el capitalismo del shock, porque como lo ha dicho el Obispo Héctor Epalza, "El puerto se convirtió en la patria del miedo y mientras el progreso avanza, el genocidio continúa ". Nada debe detener el libre comercio, así que mientras se pica a la gente con motosierra en los barrios de bajamar, los negocios andan boyantes, porque los barcos entran y salen del Puerto llevando y trayendo mercancias de toda clase, entre ellas armas y cocaína, que tanto le fascinan a los Estados Unidos, el campeón mundial de la guerra y de la imposición del libre mercado.

"De rodillas, escondido detrás de un precario horno de leña, Ismael Peña vio la forma como sus vecinos eran asesinados. El campesino, de 35 años de edad, guardó en lo más profundo de su alma el dolor que sentía al presenciar que los hombres armados con fusiles, machetes y motosierras, humillaban a sus víctimas. [...] Los hombres armados primero insultaban a las personas. Les decían que eran guerrilleros mal nacidos y que iban a morir como perros". 
El País (Cali), abril 16 del 2001.

"Es lo peor: desmembrar a las personas. Y eso no es imaginación, eso es una vergüenza. Esa es la barbarie de hasta dónde ha llegado la violencia en Buenaventura [...] Buenaventura es el corredor estratégico para la salida de la droga [...]. Pero no es sólo la droga. Hay muchos otros intereses [...]. Los megaproyectos también han azuzado la violencia". (Héctor Epalza, Obispo de Buenaventura, BBC Mundo, 24 de marzo de 2014). 


\section{Referencias bibliográficas.}

Almario, O. (2007). "ay mi bello puerto del mar, mi Buenaventura". Revista de la Universidad del Valle, 15-18.

Balbin , J., \& Insuasty Rodriguez, A. (2010). Las Victimas en Contextos de Violencia e Impunidad: Caso Medellin. Medellin: Instituto Popular de Capacticación.

Balbin, J., \& Insuasty Rodriguez, A. (2009). Victimas, Violencia y Despojo. Medellín: Litoimpacto.

Bastidas, W., \& Insuasty Rodriguez, A. (2010). Victimas en Medellín. EL AGORA USB, 367-397.

Brecht, B. (1994). Diálogos de refugiados. Madrid: Alianza Editorial.

Contreras, H. (30 de Noviembre de 1999). Palma africana en el Chocó: una nueva plaga para indios y negros. Obtenido de actualidadetnica.com: http://actualidadetnica.com/sitioNuevo/actualidad/actualidadcol-01/medio-ambiente /2494-palma-africana-en-el-choco-unanueva-plaga-para-indios-y-negros

Davis, M. (2007). Planeta de ciudades meseria. Madrid: La Foca.

E1 Espectador. (28 de marzo de 2014). Montería ganó premio como la 'Ciudad Sostenible del Planeta'. Obtenido de www.elespectador.com: http:// www.elespectador.com/noticias/medio-ambiente/monteria-ganopremio-ciudad-sostenible-del-planeta-articulo-483480

El Espectador. (17 de Abril de 2014). Tras el Foro Urbano, reaparecen habitantes de la calle en Medellin. Obtenido de El Espectador: http:// www.elespectador.com/noticias/nacional/tras-el-foro-urbanoreaparecen-habitantes-de-calle-mede-articulo-487480

El Tiempo. (25 de abril de 2014). Uribe pide que se investigue si tiene nexos con 'los Urabeños'. Obtenido de www.eltiempo.com: http://www.eltiempo. com/politica/ARTICULO-WEB-NEW_NOTA_INTERIOR-13881116.html

Franco Restrepo, V. L. (2011). Medellín, orden, desigualdad, fragilidad. Medellín: Corporación Jurídica Libertad.

Insuasty Rodriguez, A., Grisales Grajales, D., \& Gutierrez Leon, E. M. (30 de Diciembre de 2013). Conflictos asociados a la gran mineria en Antioquia. El Agora USB, 13(2), 371-397. Obtenido de http://web. usbmed.edu.co/usbmed/elagora/htm/v13nro2/pdf/CONFLICTOSASOCIADOS-A-LA-GRAN-MINERIA.pdf 
Insusty Rodriguez, A., \& Vallejo Duque, Y. (2008). ACCIÓN SOCIAL ¿UNA DINÁMICA PARA EL DESARROLLO SOCIAL O UNA ESTRATEGIA PARA EL CONTROL TERRITORIAL? EL AGORA USB, 101-122.

Klein, N. (2007). La doctrina del Shock. El auge del capitalismo del desastre. Barcelona: Editorial Paidós.

Legiscomex.com. (9 de Abril de 2014). La problemática en Buenaventura: más allá de la droga, el contrabando y las Bacrim. Obtenido de legiscomex. com: http://www.legiscomex.com/BancoConocimiento/P/ problematica-buenaventura-afectacion-comercio-puerto-rci284/ problematica-buenaventura-afectacion-comercio-puerto-rci284. asp?CodSubseccion $=389 \&$ numarticulo $=\&$ CodSeccion $=190$

Molano Jimeno, A. (23 de Febrero de 2013). Buenaventura, entre la pobreza y la miseria. Obtenido de El Espectador: http://www.elespectador. $\mathrm{com} /$ noticias / nacional/ buenaventura-entre-pobreza-y-violenciaarticulo-406499

O Loingsigh, G. (2013). La reconquista del paciífico. Invasión, inversión, impunidad. Bogotá: Proceso de comunidades Negras.

Revista Semana. (5 de Junio de 2005). Habla Vicente Castaño. Obtenido de Revista Semana: http://www.semana.com/portada/articulo/hablavicente-castano/72964-3

Revista Semana. (31 de Julio de 2006). El 'Führer' de Urabá. Obtenido de Verdad Abierta: http://www.verdadabierta.com/victimarios/losjefes /308-el-fuehrerde-uraba

Revista Semana. (18 de Enero de 2011). Piden demoler monumento a paramilitares en Montería. Obtenido de www.semana.com: http:// www.semana.com/nacion/articulo/piden-demoler-monumentoparamilitares-monteria/234274-3

Salazar, B. (Julio de 2007). "Morir en Buenaventura: entre el gueto y la prisión”. Revista de la Universidad del Valle., 1, 58.

Valenzuela, S. (5 de abril de 2014). Destierro en Baja Mar. Obtenido de El Espectador: http://www.elespectador.com/noticias/nacional/ destierro-bajamar-articulo-485127

Vallejo Duque, Y., \& Insuasty Rodriguez, A. (2009). Desarrollo Social Para el Control territorial. Circulo de Humanidades, 99-110. 
Verdad Abierta. (28 de Marzo de 2014). Ex jefe del Bloque Élmer Cárdenas de las Auc que operó en el Urabá. Obtenido de verdad abierta: http:// www.verdadabierta.com/victimarios / perfiles-de-paramilitares/431a/716-perfil-freddy-rendon-herrera-alias-el-aleman

Weis, S. (14 de Junio de 2012). Buenaventura. La puerta del "Chapo" en Colombia. Obtenido de contrapunto.com.sv: http://www.contrapunto. com.sv/latinoamerica/buenaventura-la-puerta-del-chapo-en-colombia 